\title{
Health benefits of electrically-assisted cycling: a systematic review
}

\author{
Jessica E. Bourne ${ }^{1,2^{*}}$ (D), Sarah Sauchelli ${ }^{2}$, Rachel Perry ${ }^{2}$, Angie Page ${ }^{1,2}$, Sam Leary ${ }^{2}$, Clare England ${ }^{1,2}$ \\ and Ashley R. Cooper ${ }^{1,2}$
}

\begin{abstract}
Background: Electrically assisted bicycles (e-bikes) have been highlighted as a method of active travel that could overcome some of the commonly reported barriers to cycle commuting. The objective of this systematic review was to assess the health benefits associated with e-cycling.

Method: A systematic literature review of studies examining physical activity, cardiorespiratory, metabolic and psychological outcomes associated with e-cycling. Where possible these outcomes were compared to those from conventional cycling and walking. Seven electronic databases, clinical trial registers, grey literature and reference lists were searched up to November 2017. Hand searching occurred until June 2018. Experimental or observational studies examining the impact of e-cycling on physical activity and/or health outcomes of interest were included. Ebikes used must have pedals and require pedalling for electric assistance to be provided.

Results: Seventeen studies (11 acute experiments, 6 longitudinal interventions) were identified involving a total of 300 participants. There was moderate evidence that e-cycling provided physical activity of at least moderate intensity, which was lower than the intensity elicited during conventional cycling, but higher than that during walking. There was also moderate evidence that e-cycling can improve cardiorespiratory fitness in physically inactive individuals. Evidence of the impact of e-cycling on metabolic and psychological health outcomes was inconclusive. Longitudinal evidence was compromised by weak study design and quality.

Conclusion: E-cycling can contribute to meeting physical activity recommendations and increasing physical fitness. As such, e-bikes offer a potential alternative to conventional cycling. Future research should examine the long-term health impacts of e-cycling using rigorous research designs.
\end{abstract}

Keywords: Electrically-assisted bicycle, E-bike, Physical activity, Health

\section{Background}

Given the high rates of global physical inactivity [1] a growing body of research has focused on the potential of active travel to increase physical activity behaviour and potentially lead to population health benefits. Engagement in active travel, specifically commuting, has been shown to be predictive of a lower BMI [2] and reduced risk of diabetes diagnosis [3]. A recent prospective study reported that active commuting, involving cycling, was associated with a lower risk of all-cause mortality and cancer incidence and mortality [4]. In addition, commuting by bicycle or on foot was

\footnotetext{
* Correspondence: jessica.bourne@bristol.ac.uk

${ }^{1}$ Centre for Exercise, Nutrition and Health Sciences, School of Policy Studies, University of Bristol, 8 Priory Road, Bristol BS8 1TZ, UK

${ }^{2}$ NIHR Bristol Biomedical Research Centre, University Hospitals Bristol NHS Foundation Trust and University of Bristol, Bristol, UK
}

associated with a lower risk of cardiovascular disease incidence and mortality [4]. The greatest gains in health outcomes from active commuting are reported in the least active individuals $[5,6]$.

Travel is an essential part of everyday life for most people, and the adoption of active travel represents an efficient way to increase daily physical activity. For example, Falconer and colleagues [2] found that active commuting was associated with an additional 73 weekly minutes of moderate to vigorous physical activity in men and 105 weekly minutes in women with type 2 diabetes, compared to those commuting using motorised transport. With half of all car journeys in the UK being between 1 and 5 miles in length [7], the substitution of many car journeys by walking and/or cycling may be an achievable aim. 
Due to a growing body of evidence, the UK National Institute of Health and Care Excellence (NICE) now endorse active travel, with a particular focus on commuting, as a feasible method to incorporate physical activity into daily life [8]. However, rates of active commuting are low [9].Common barriers to cycle commuting include the physical constraints associated with hilly terrain, poor physical fitness, lack of time and the distance to work [10].

Electrically assisted bicycles (e-bikes) have been highlighted as an alternative method of active travel that could overcome some of the commonly reported barriers to cycle commuting [11]. The term e-bike includes a range of designs including throttle-controlled bikes which do not require the rider to pedal and electrically assisted bikes which provide electrical assistance only when the rider is pedalling, through sensors which detect pedalling speed and force [11]. It is through pedalling that electrically-assisted cycling may serve to increase physical activity. With lower motor power and maximum speeds compared to throttle-controlled e-bikes, electrically-assisted bikes are legally classified as bicycles. [11]. For this review the term e-bike will be used exclusively to refer to electrically-assisted bicycles which require the rider to pedal.

In recent years e-bikes have become commonplace in European countries [11] with projected global sales of 47.6 million by the end of 2018 [12]. E-bikes are increasingly used for both leisure and commuting purposes [13]. The assistance provided has been reported to motivate novice cyclists and increase the likelihood that these individuals will continue to cycle in the future [10]. Given the increasing interest in e-bikes, and their use for active travel, there is a need to understand their potential to promote physical activity of a sufficient intensity to gain clinical benefit (i.e., moderate-to-vigorous intensity [14]) and to examine their impact on broader health outcomes. Such research is required to inform relevant health economic assessments and public health policy. To date, there has been no systematic review on the physical activity intensity and health outcomes associated with e-cycling. As such the aims of this systematic review are to answer the following research questions:

1. What is the intensity of physical activity associated with riding an e-bike?

2. Does use of an e-bike lead to changes in health outcomes including cardiorespiratory, metabolic or psychological outcomes?

3. Do physiological responses to riding an e-bike differ to those generated by other modes of active transportation (i.e. walking and conventional cycling)?

\section{Methods}

A review protocol was registered at the PROSPERO database: Registration number CRD42018086544 (http://www.crd.york.ac.uk/prospero). This review was conducted according to the guidelines outlined by the Preferred Reporting Items for Systematic Reviews and Meta-Analyses guidelines [15].

\section{Search strategy}

The following databases were searched from their inception to November 2017: PsychINFO, MEDLINE and Embase (via Ovid), ISI Web of Science, CINAHL complete, SPORTDiscus and Scopus. Search terms were 'pedelec', 'e-bike' 'electrically assisted bicycle, 'electrically assisted cycle', 'electrically assisted bike,' pedal-assist,'electric bicycle,'electric bike,'electric cycle,'electric mobility' (see Additional file 1 for example). Reference lists from all selected articles were hand-searched for relevant studies. OpenGrey and Google Scholar (first 20-pages) were searched using the term 'electrically-assisted bicycle'. Hand-searching occurred until June 2018.

\section{Inclusion criteria and selection process}

Studies were eligible for inclusion if they met the following criteria:

1) participants: adults $\geq 18$ years of age,

2) electrically-assisted bicycle must have pedals and be operated by the individual, with assistance available from an electric motor

3) at least one of the following outcomes; objective measure of physical activity intensity whilst ecycling (e.g., metabolic equivalents, energy expenditure), cardiorespiratory, metabolic or quality of life (as a measure of psychological health),

4) type of study: experimental or observational studies.

Studies could be published or unpublished in any language. For articles in a language other than English the title and abstract were translated using Google Translate. If full text screening was required, the article was translated by an individual fluent in the language. Studies were excluded if they reported using bicycles that did not require the individual to pedal to provide power, were review articles or commentary pieces, and/or used self-reported measures of physical activity. Title and abstract screening was conducted by two reviewers independently (J.E.B. and S.S.). There was a 93\% agreement between reviewers on title and abstract screening. Full texts were screened by the two reviewers independently and any discrepancies were discussed.

\section{Quality assessment and strength of the evidence}

The quality of included studies was assessed using the Quality Assessment Tool for Quantitative Studies (EPHPP; [16]). The tool appraises studies on six components; 1) selection bias, 2) study design, 3) control of confounders, 4) blinding, 5) reliability and validity of data collection methods and 6) withdrawals and dropouts. Each component was rated as; strong, moderate or weak for each study based on outcomes of interest. 
A global rating for each study was then determined based on the criteria; 1 ) strong when no weak ratings were reported, 2) moderate when one weak rating was reported, and 3) weak when two or more components were rated as weak. This tool has been used in a previous review examining the impact of cycling on health [6]. The blinding component was not included in the overall study rating as participants are unable to be blinded to condition allocation following randomisation in physical activity interventions. The overall strength of the evidence was assessed based on previously specified best evidence synthesis criteria [17] (Additional file 2).

\section{Data extraction and synthesis}

Members of the review team (J.E.B and either S.S. or A.R.C) independently extracted data for each study. Quality assessment was confirmed by a fourth reviewer (R.P.). Data were extracted using an adapted version of a Cochrane Data Extraction Form, which was piloted prior to use. Discrepancies regarding data extraction were resolved through discussion between reviewers. Data extracted included study design, characteristics of participants, outcomes measured, and results. Due to the heterogeneity of study design and outcomes reported, a meta-analysis was not deemed appropriate. Data were synthesized and presented narratively. The effect of the intervention on physical activity and health outcomes for each study was summarized based on reported statistical significance and effect size, both within group (pre-post) and between group where possible, or by examining means or medians when no hypothesis testing was conducted.

\section{Results}

A total of 4399 articles were identified through initial searches (Fig. 1). After removing duplicates 2894 titles and abstracts were screened, resulting in 119 studies which underwent full text screening for inclusion. Sixteen articles met the criteria for inclusion plus one included after author contact. Eleven studies assessed the acute response to e-cycling (i.e., one bout of e-cycling), and six examined the longitudinal effect of e-cycling (i.e., more than one bout of e-cycling, including pre-post measurements). Reasons for exclusion included no measure of specified outcomes, study not related to e-bikes, studies focused on the engineering of e-bikes, qualitative studies or not presenting original research. Three studies were identified through clinicaltrials.gov but were excluded for the following reasons: 1) data not published, 2) currently recruiting, 3) authors were not reachable.

\section{Study characteristics}

\section{Acute studies}

Eleven studies examined the acute physiological impact of e-cycling using cross over designs, five of which were randomized (Table 1). Nine studies were conducted in Europe and two in the USA. Sample sizes ranged from 3 to 22 with a total of 147 participants. Participants were aged between 20 and 70 . Three studies recruited physically inactive individuals [18-20] and one study included individuals with coronary artery disease [21]. Six studies compared e-cycling to conventional cycling [18, 21-25] and five compared e-cycling with assist to riding an e-bike without assistance [19, 20, 26-28]. Two studies included walking as a comparator $[18,23]$.

Rest periods between conditions ranged from 2-min to 1 month and distance ridden from 3.54 to $27 \mathrm{~km}$. Nine studies were conducted in a natural setting with topography ranging from flat to elevations between 33.5 and $260 \mathrm{~m}$. Four studies specifically examined the impact of topography on physiological outcomes by separating rides into different topographical sections (Additional file 3). Four studies required participants to stop and go during rides to simulate typical riding conditions $[20,26]$ or delivering mail $[24,25]$. In seven studies participants were instructed to ride at a self-selected pace.

\section{Longitudinal studies}

Six studies examined the longitudinal impact of e-cycling, using a variety of study designs (Table 1 ). All studies were conducted in high income countries including Belgium, Switzerland, Norway, UK $(n=2)$ and the USA. Sample sizes ranged from 20 to 32, with a total of 153 participants. Most participants were between 30 and 50 years of age. Four studies recruited physically inactive individuals [13, 29-31]. One study included individuals with type 2 diabetes [32] and for one study the health status of individuals was unclear [33]. Interventions ranged from 4-weeks to 8-months in length. One study included published data from mid-point of the intervention, but not post intervention [33]. Three studies provided participants with guidelines on minimum riding requirements, all of which specified riding the e-bike for commuting purposes at least three times per week $[13,29,30]$.

\section{Physical activity intensity}

Studies reported a range of outcomes related to physical activity intensity. Given the heterogeneity between studies regarding route length and topography, mean values and/or percent of maximum values during conditions are reported to enable comparison between studies. Physiological outcomes reported within the manuscript include oxygen uptake, metabolic equivalents, ${ }^{1}$ energy expenditure per minute, heart rate and power output (Table 2). Additional outcomes are reported in Additional file 4 and Additional file 5 . 


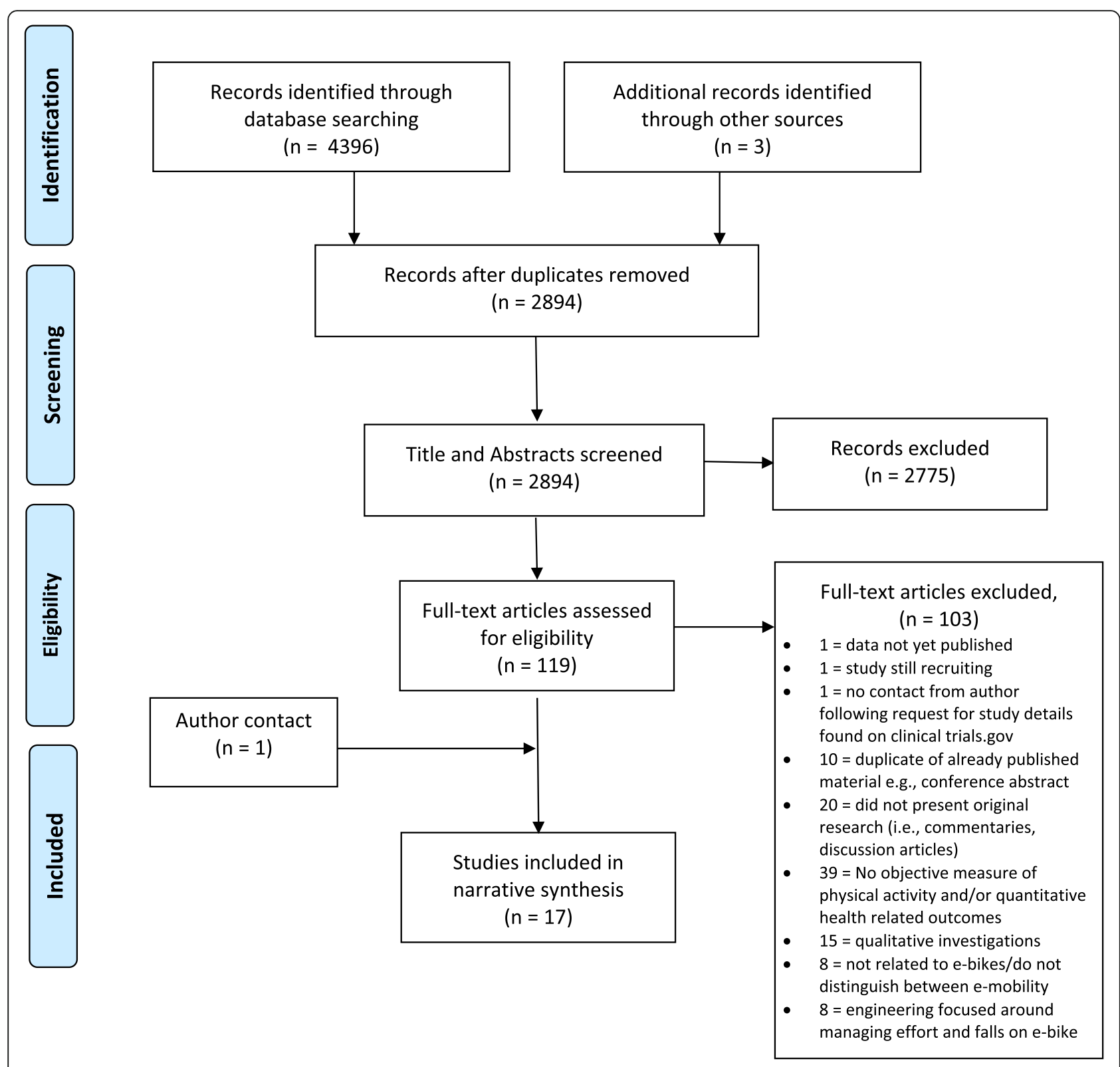

Fig. 1 Flow chart of literature search

\section{Oxygen uptake}

Eight studies reported oxygen uptake [18, 19, 21-23, 25-27]. Riding an e-bike led to a relative mean oxygen uptake of 14.7 to $29 \mathrm{ml} / \mathrm{min} / \mathrm{kg}$ or 51 to $74 \%$ of maximum oxygen uptake. E-cycling required lower oxygen uptake than conventional cycling (19.3 to 37 $\mathrm{ml} / \mathrm{min} / \mathrm{kg}$ ) or e-cycling with no assistance (22.9 to $23.4 \mathrm{ml} / \mathrm{min} / \mathrm{kg}$ ), with statistically significant differences reported in four studies, one of which reported an effect size of 1.73 [19]. Walking elicited lower oxygen uptake compared to self-selected e-cycling [23] and e-cycling on low assist [18].

\section{Metabolic equivalents (METs)}

Nine studies reported mean estimated METs while riding an e-bike at a self-selected intensity [13, 1823, 26, 27], which ranged from 4.9 to 8.3 METs. Overall, e-cycling led to a lower mean MET score than conventional cycling or e-cycling without assistance. However, the significance of the difference is inconclusive. One study reported a difference in mean METs between walking and e-cycling only during uphill sections [23], while another study reported no difference between walking and e-cycling over varied terrain [18]. 
Table 1 Summary of included studies

\begin{tabular}{|c|c|c|c|c|c|c|}
\hline $\begin{array}{l}\text { First author, } \\
\text { year, country }\end{array}$ & Study design & $\begin{array}{l}\text { Participants; } \\
\text { gender } \\
\text { (\%female); } \\
\text { Age, years } \\
\text { (mean, SD); } \\
\text { BMl, kg/m² } \\
\text { (mean, SD) }\end{array}$ & Clinical status & $\begin{array}{l}\text { Exposure } \\
\text { conditions }\end{array}$ & Length of intervention & $\begin{array}{l}\text { Ride characteristics } \\
\text { Distance }(\mathrm{km}) \text {, Topography, Distinctive } \\
\text { features, Ride instructions }\end{array}$ \\
\hline
\end{tabular}

\begin{tabular}{|c|c|c|c|c|c|c|}
\hline \multicolumn{7}{|l|}{ Acute studies } \\
\hline $\begin{array}{l}\text { Bernsten, } \\
\text { 2017, } \\
\text { Norway [22] }\end{array}$ & $\begin{array}{l}\text { Randomized } \\
\text { cross over }\end{array}$ & $\begin{array}{l}N=8,25 \% \\
\text { Age }(M d n, I Q R): \\
39(3) \\
\text { BMI }(M d n, I Q R): \\
24(7)\end{array}$ & Active adults & $\begin{array}{l}\text { E-bike vs. CB } \\
\text { ( } 4 \text { conditions, } \\
\text { hilly vs. flat } \\
\text { terrain) }\end{array}$ & $\begin{array}{l}\text { Trials conducted on } \\
\text { same day, 2-min break } \\
\text { between trials }\end{array}$ & $\begin{array}{l}\text { Route 1: } 8.1 \mathrm{~km} \text {, flat route } \\
\text { Route 2: } 7.1 \mathrm{~km} \text {, one hill climbed twice } \\
130 \mathrm{~m} \text { elevation gain. Self-selected } \\
\text { intensity }\end{array}$ \\
\hline $\begin{array}{l}\text { Gojanovic, } \\
2011, \\
\text { Switzerland } \\
{[18]}\end{array}$ & $\begin{array}{l}\text { Non- } \\
\text { randomized } \\
\text { cross over }\end{array}$ & $\begin{array}{l}N=18,33.33 \% \\
\text { Age: } 35.7( \pm 9.7) \\
\text { BMl: } 24.0( \pm 3.3)\end{array}$ & Inactive adults & $\begin{array}{l}\text { E-bike LA vs. } \\
\text { E-bike HA vs. } \\
\text { CB vs. } \\
\text { walking }\end{array}$ & $\begin{array}{l}\text { Trials conducted over } \\
\text { 2-days. 30-min break } \\
\text { between trials con- } \\
\text { ducted on same day }\end{array}$ & $\begin{array}{l}\text { Biking: } 5.1 \mathrm{~km}, 178 \mathrm{~m} \text { elevation gain, } \\
\text { average gradient } 3.4 \% \text { Instructed to ride } \\
\text { at comfortable pace maintaining } 60 \mathrm{rpm} \\
\text { Walking: } 1.7 \mathrm{~km} \text {, uphill, } 110 \mathrm{~m} \text { elevation } \\
\text { gain, average grade } 6.5 \%\end{array}$ \\
\hline $\begin{array}{l}\text { Hansen, } \\
\text { 2017, } \\
\text { Belgium [21] }\end{array}$ & $\begin{array}{l}\text { Randomized } \\
\text { cross over }\end{array}$ & $\begin{array}{l}N=17,13 \% \\
\text { Age: } 64( \pm 7)\end{array}$ & $\begin{array}{l}\text { Coronary } \\
\text { artery disease }\end{array}$ & $\begin{array}{l}\text { E-bikes LA } \\
\text { vs. E-bike HA } \\
\text { vs. CB }\end{array}$ & $\begin{array}{l}\text { Trials conducted on } \\
\text { separate days ( } 3-4 \text { days } \\
\text { between) }\end{array}$ & $\begin{array}{l}10 \mathrm{~km}, 102 \mathrm{~m} \text { elevation change } \\
\text { No traffic or stop and go points } \\
\text { Instructed to cycle at self-selected pace } \\
\text { on prespecified mode }\end{array}$ \\
\hline $\begin{array}{l}\text { La Salle, } \\
\text { 2017, USA } \\
{[26]}\end{array}$ & $\begin{array}{l}\text { Randomized } \\
\text { cross over }\end{array}$ & $\begin{array}{l}N=12,50 \% \\
\text { Age: } M=25( \pm 1) \\
F=22( \pm 1) \\
\text { Body Fat } \%: M \\
=16.8( \pm 1.9), F= \\
23.4( \pm 3.3)\end{array}$ & $\begin{array}{l}\text { Active adults } \\
\text { with cycling } \\
\text { experience }\end{array}$ & $\begin{array}{l}\text { E-bike pedal } \\
\text { assist vs. E- } \\
\text { bike NA }\end{array}$ & $\begin{array}{l}\text { Trials conducted in } \\
\text { same day. Average } \\
\text { time between trials 12- } \\
\text { min }\end{array}$ & $\begin{array}{l}3.54 \mathrm{~km} \text {, hill } 0.64 \mathrm{~km} 11 \% \text { gradient } \\
\text { Seven pedestrian crossings participants } \\
\text { required to dismount and walk. Self- } \\
\text { selected pace }\end{array}$ \\
\hline $\begin{array}{l}\text { Langford, } \\
\text { 2017, USA } \\
\text { [23] }\end{array}$ & $\begin{array}{l}\text { Non- } \\
\text { randomized } \\
\text { cross over }\end{array}$ & $\begin{array}{l}\mathrm{N}=17,35 \% \\
\text { Age: }<20 \mathrm{yrs} .= \\
3,20-30 \mathrm{yrs} .=10, \\
31-40 \text { yrs. }=2,> \\
50 \text { yrs. }=2 \\
\text { BMI: } M=26.1, \mathrm{~F} \\
=23.1\end{array}$ & $\begin{array}{l}\text { Adults, part of } \\
\text { e-bike sharing } \\
\text { system }\end{array}$ & $\begin{array}{l}\text { E-bike vs. CB } \\
\text { vs. Walking }\end{array}$ & $\begin{array}{l}\text { Trials conducted on } \\
\text { separate days } \\
\text { (minimum 24h rest) }\end{array}$ & $\begin{array}{l}4.4 \mathrm{~km}, 1.6 \mathrm{~km} \text { downhill }(-33.2 \mathrm{~m}), 1.8 \mathrm{~km} \\
\text { flat }(-0.3 \mathrm{~m}), 1.0 \mathrm{~km} \text { uphill }(+33.5 \mathrm{~m}) . \text { Self- } \\
\text { selected pace }\end{array}$ \\
\hline $\begin{array}{l}\text { Louis, 2012, } \\
\text { France [27] }\end{array}$ & $\begin{array}{l}\text { Randomized } \\
\text { cross-over }\end{array}$ & $\begin{array}{l}N=20(10 T, 10 \\
\text { UT) } \\
\text { Age: } T=38.7 \\
( \pm 14.8) ; \text { UT } 28.9 \\
( \pm 6.3) \\
\text { BMI: T }=22 \\
( \pm 1.1), \text { UT }=22.2 \\
( \pm 3.7)\end{array}$ & $\begin{array}{l}\text { Highly active } \\
\text { adults }(T) \\
\text { Recreationally } \\
\text { active adults } \\
\text { (UT) }\end{array}$ & $\begin{array}{l}\text { E-bike NA } \\
\text { vs. E-bike LA } \\
\text { vs. E-bike HA }\end{array}$ & $\begin{array}{l}\text { Trials conducted on } \\
\text { same day. 5-min breaks } \\
\text { between trials }\end{array}$ & $\begin{array}{l}\text { Completed on indoor trainer. } \\
\text { Instructed to pedal at specified mode for } \\
\text { total of } 45-\text { min at pre-specified speeds: } \\
15 \text {-min at } 16 \mathrm{~km} / \mathrm{hr} \text {., } 21 \mathrm{~km} / \mathrm{hr} \text {. and free } \\
\text { speed totaling } 45-\text { min. }\end{array}$ \\
\hline $\begin{array}{l}\text { Meyer, 2014, } \\
\text { Germany } \\
{[28]}\end{array}$ & $\begin{array}{l}\text { Non- } \\
\text { randomized } \\
\text { cross over }\end{array}$ & $\begin{array}{l}N=3,0 \% \\
\text { Age: } 25,25,27 \\
\text { Weight }(\mathrm{Kg}): 74 \\
71,79\end{array}$ & $\begin{array}{l}\text { Active adults, } \\
\text { recreational } \\
\text { cyclists }\end{array}$ & $\begin{array}{l}\text { E-bike pedal } \\
\text { assist vs. E- } \\
\text { bike no } \\
\text { assist }\end{array}$ & $\begin{array}{l}\text { Trials conducted on } \\
\text { separate days, 1-day } \\
\text { apart. }\end{array}$ & $27 \mathrm{~km}$ track divided in 5 sections \\
\hline $\begin{array}{l}\text { Simons, } \\
\text { 2009, } \\
\text { Netherlands } \\
{[20]}\end{array}$ & $\begin{array}{l}\text { Non- } \\
\text { randomized } \\
\text { cross over }\end{array}$ & $\begin{array}{l}N=12,50 \% \\
\text { Age: } 52.2(8.7), \\
\text { range } 32-60 \\
\text { BMl: } 24.5(2.6)\end{array}$ & $\begin{array}{l}42 \% \text { inactive } \\
\text { adults } \\
58 \% \\
\text { recreationally } \\
\text { active adults }\end{array}$ & $\begin{array}{l}\text { E-bike NA } \\
\text { vs. E-bike LA } \\
\text { vs. E-bike HA }\end{array}$ & $\begin{array}{l}\text { Trials conducted in } \\
\text { same day. One-hour } \\
\text { rest between trials. }\end{array}$ & $\begin{array}{l}4.3 \mathrm{~km} \text {, flat route, two stop and go section } \\
\text { participants required to dismount and } \\
\text { restart. Self-selected pace on pre-specified } \\
\text { intensity }\end{array}$ \\
\hline $\begin{array}{l}\text { Sperlich, } \\
\text { 2012, } \\
\text { Germany } \\
{[19]}\end{array}$ & $\begin{array}{l}\text { Randomized } \\
\text { cross over }\end{array}$ & $\begin{array}{l}N=8,100 \% \\
\text { Age: } 38( \pm 15) \\
\text { BMl: } 25.3( \pm 2.1)\end{array}$ & Inactive adults & $\begin{array}{l}\text { E-bike pedal } \\
\text { assist vs. E- } \\
\text { bike no } \\
\text { assist }\end{array}$ & $\begin{array}{l}\text { Trials conducted in } \\
\text { same day. One-hour } \\
\text { rest between trials. }\end{array}$ & $\begin{array}{l}1.9 \mathrm{~km} \times 5=9.5 \mathrm{~km}, 200 \mathrm{~m} \text { uphill 1,5.9\%, } \\
700 \mathrm{~m} \text { downhill, } 300 \mathrm{~m} \text { uphill 2, 5.8\%, } 700 \\
\mathrm{~m} \text { flat. Self-selected pace and gear }\end{array}$ \\
\hline $\begin{array}{l}\text { Theurel, } \\
\text { 2011, France } \\
\text { [24] }\end{array}$ & $\begin{array}{l}\text { Non- } \\
\text { randomized } \\
\text { cross over }\end{array}$ & $\begin{array}{l}N=22,18 \% \\
\text { female } \\
\text { Age: } M= \\
41( \pm 11), F= \\
34( \pm 9) \\
\text { Weight }(\mathrm{Kg}): \mathrm{M} \\
=68( \pm 18), \mathrm{F}= \\
76( \pm 10)\end{array}$ & $\begin{array}{l}\text { Active postal } \\
\text { workers }\end{array}$ & E-bike vs. CB & $\begin{array}{l}\text { Trials conducted on } \\
\text { same weekday, 1- } \\
\text { month apart }\end{array}$ & $\begin{array}{l}\text { Postal route, one group completed rides } \\
\text { in residential neighbourhood, the other } \\
\text { completed the ride in downtown location }\end{array}$ \\
\hline
\end{tabular}


Table 1 Summary of included studies (Continued)

\begin{tabular}{|c|c|c|c|c|c|c|}
\hline $\begin{array}{l}\text { First author, } \\
\text { year, country }\end{array}$ & Study design & $\begin{array}{l}\text { Participants; } \\
\text { gender } \\
\text { (\%female); } \\
\text { Age, years } \\
\text { (mean, SD); } \\
\text { BMl, kg/m² } \\
\text { (mean, SD) }\end{array}$ & Clinical status & $\begin{array}{l}\text { Exposure } \\
\text { conditions }\end{array}$ & Length of intervention & $\begin{array}{l}\text { Ride characteristics } \\
\text { Distance }(\mathrm{km}) \text {, Topography, Distinctive } \\
\text { features, Ride instructions }\end{array}$ \\
\hline $\begin{array}{l}\text { Theurel, } \\
\text { 2012, France } \\
\text { [25] }\end{array}$ & $\begin{array}{l}\text { Non- } \\
\text { randomized } \\
\text { cross over }\end{array}$ & $\begin{array}{l}N=10,50 \% \\
\text { female } \\
\text { Age: } F=30 \\
( \pm 12), M=35 \\
( \pm 14)\end{array}$ & Active adults & E-bike vs. CB & $\begin{array}{l}\text { Trials separated by } 1 \\
\text { week }\end{array}$ & $\begin{array}{l}\text { 30-min of intermittent cycling on inside } \\
\text { track alternating cycling of } 10 \mathrm{sec} \text { duration } \\
\text { and recovery of } 20 \mathrm{sec} \text {. Aimed to } \\
\text { complete } 60 \mathrm{~m} \text { in } 10 \mathrm{sec} \text { (average speed } \\
=21.6 \mathrm{~km} / \mathrm{hr} \text { ) }\end{array}$ \\
\hline \multicolumn{7}{|c|}{ Longitudinal studies } \\
\hline $\begin{array}{l}\text { Cooper, } \\
\text { 2018, UK [32] }\end{array}$ & $\begin{array}{l}\text { Single group } \\
\text { feasibility }\end{array}$ & $\begin{array}{l}N=20 \text { (report } \\
\text { on 18) } \\
\text { Age: } 58.1( \pm 7.9) \\
\text { BMI: } 30.2(4.4)\end{array}$ & $\begin{array}{l}\text { Type } 2 \\
\text { Diabetes }\end{array}$ & $\begin{array}{l}\text { One group } \\
\text { e-bike }\end{array}$ & Up to 5 months & $\begin{array}{l}\text { E-bike training provided. Provision of e- } \\
\text { bike for up to } 5 \text {-months. Support for } \\
\text { mechanical issues provided. No instruc- } \\
\text { tion on how or when to ride bike }\end{array}$ \\
\hline $\begin{array}{l}\text { De Geus, } \\
\text { 2013, } \\
\text { Belgium [29] }\end{array}$ & $\begin{array}{l}\text { Non- } \\
\text { randomized } \\
\text { cross over }\end{array}$ & $\begin{array}{l}N=24,46 \% \\
\text { Age: } M=47( \pm 7) \\
F=43( \pm 6) \\
\text { BMl: } M=27.0 \\
( \pm 2.8), F=24.7 \\
( \pm 4.6)\end{array}$ & $\underset{a}{\text { Inactive adults }}$ & $\begin{array}{l}\text { E-bike vs. } \\
\text { Control }\end{array}$ & $\begin{array}{l}\text { Control }=4 \text { weeks } \\
\text { E-bike }=6 \text { weeks }\end{array}$ & $\begin{array}{l}\text { Instructed to ride e-bike at least three } \\
\text { times per week to commute to and from } \\
\text { work }\end{array}$ \\
\hline $\begin{array}{l}\text { Hochsmann, } \\
2017 \text {, } \\
\text { Switzerland } \\
{[30]}\end{array}$ & $\begin{array}{l}\text { Pilot } \\
\text { randomized } \\
\text { controlled } \\
\text { trial }\end{array}$ & $\begin{array}{l}N=32,13 \% \\
\text { Age, }(M d n, I Q R): \\
F=35(34-45) \\
M=43(38-45) \\
\text { BMl, }(M d n, I Q R) \\
\text { E-bike }=29 \\
(27,31), \text { regular } \\
\text { bike }=28(26,29)\end{array}$ & Inactive adults & E-bike vs. CB & 4 weeks & $\begin{array}{l}\text { Instructed to use bike for active commute } \\
\text { to work on at least 3-days per week, over } \\
6 \mathrm{~km} \text {. Self-selected pace }\end{array}$ \\
\hline $\begin{array}{l}\text { Malnes, } \\
\text { 2016, } \\
\text { Norway [31] }\end{array}$ & $\begin{array}{l}\text { Single group } \\
\text { pilot }\end{array}$ & $\begin{array}{l}N=25,72 \% \\
\text { Age: } 42( \pm 12) \\
\text { BMl: } M= \\
25.4( \pm 12.3), F= \\
28.7( \pm 15.8)\end{array}$ & Inactive adults & $\begin{array}{l}\text { One group } \\
\text { e-bike }\end{array}$ & Up to 8 months & $\begin{array}{l}3 \text { sites: } 2 \text { provided e-bikes for up to } 8 \text { - } \\
\text { months, } 1 \text { e-bike up to 3-months. } \\
\text { Instructed to use bike as desired. In 2- } \\
\text { centres if e-bikes not used they were } \\
\text { withdrawn from participant. Group was } \\
\text { separated into high and low fitness } \\
\text { groups based on baseline testing }\end{array}$ \\
\hline $\begin{array}{l}\text { Page, 2017, } \\
\text { UK [33] }\end{array}$ & $\begin{array}{l}\text { Non- } \\
\text { randomized } \\
\text { two group }\end{array}$ & $\begin{array}{l}N=31,80 \% \\
\text { Age Range: } 21- \\
55 \text { years }\end{array}$ & Unclear & $\begin{array}{l}\text { E-bike } \\
\text { commuting } \\
\text { vs. passive } \\
\text { commuting }\end{array}$ & $\begin{array}{l}\text { Data reported mid-way } \\
\text { into intervention - } 2 \\
\text { months }\end{array}$ & $\begin{array}{l}\text { No instructions on how to ride bike, full } \\
\text { roadside assistance provided. }\end{array}$ \\
\hline $\begin{array}{l}\text { Peterman, } \\
\text { 2016, USA } \\
\text { [13] }\end{array}$ & Single group & $\begin{array}{l}N=21,70 \% \text { (of } \\
20 \text { in analysis) } \\
\text { Age: } 41.5 \\
( \pm 11.5)\end{array}$ & Inactive adults & $\begin{array}{l}\text { One group } \\
\text { e-bike }\end{array}$ & 4 weeks & $\begin{array}{l}\text { Instructed to ride e-bike at least } 3 \text { days } \\
\text { per week for at least } 40 \text {-min for } \\
\text { commuting }\end{array}$ \\
\hline
\end{tabular}

$T$ trained (engage in endurance sport at least 4 times per week), UT untrained (moderately active but less than $4 \times$ per week), Inactive $<150$ min/week of moderate to vigorous physical activity, Active $\geq 150 \mathrm{~min} /$ week of moderate to vigorous physical activity ${ }^{\text {a }}$ report as sedentary but do not specifically measure moderate to vigorous physical activity, $F$ female, $M$ male, $N A$ no assistance, $L A$ low assistance, $H A$ high assistance, $C B$ conventional bike

\section{Energy expenditure per minute}

Four studies assessed energy expenditure per minute $[13,23,24,27]$. On an indoor trainer, energy expenditure per minute was lower on an e-bike with assistance (high or low) compared to an e-bike without assistance in physically active adults [27]. In outdoor trials two studies reported no difference in energy expenditure per minute between e-cycling and conventional cycling, though mean values were consistently lower for e-cycling [23, 24]. Absolute energy expenditure per minute while riding an e-bike ranged from 4.9 to $6.5 \mathrm{kcal} / \mathrm{min}$.

\section{Heart rate}

Twelve studies reported heart rate while e-cycling [13, 18-20, 23-28, 30, 32]. During e-cycling the percentage of maximum heart rate ranged from 67.1 to 79.1. Overall, mean heart rate while riding an e-bike was lower than riding a conventional bike or an e-bike with no 
Table 2 Physical activity intensity outcomes of interest measured during rides ${ }^{*}$

\begin{tabular}{|c|c|c|c|c|c|c|}
\hline \multirow[t]{2}{*}{ Study } & \multirow[t]{2}{*}{ Outcomes } & \multicolumn{5}{|c|}{ Results; mean (SD) } \\
\hline & & E-bike & $\begin{array}{l}\text { Comparison } \\
1\end{array}$ & $\begin{array}{l}\text { Comparison } \\
2\end{array}$ & $\begin{array}{l}\text { Comparison } \\
3\end{array}$ & Significance testing, $p$ value \\
\hline \multirow{4}{*}{$\begin{array}{l}\text { Bernsten, } 2017 \\
{[22]^{a}}\end{array}$} & (Median, IQR) & E-bike & CB & & & \\
\hline & Percentage $\mathrm{VO}_{2 \max }$ & $51(27)$ & $58(28)$ & & & NC \\
\hline & Measured METs & $8.5(3.1)$ & $10.9(2.7)$ & & & NC \\
\hline & Estimated METs & $6.9(2.1)$ & $8.4(1.8)$ & & & NC \\
\hline \multirow[t]{5}{*}{ Cooper, 2018 [32] } & & E-bike & Walking & & & \\
\hline & Mean HR & $125.2(18.1)$ & $107.6(15.8)$ & & & NC \\
\hline & Men & $121.2(17.2)$ & $103.2(14.1)$ & & & NC \\
\hline & Women & $132.6(18.9)$ & $116.5(16.9)$ & & & NC \\
\hline & Percentage HR max & 74.7 & 64.3 & & & NC \\
\hline \multirow{6}{*}{$\begin{array}{l}\text { Gojanovic, } 2011 \\
{[18]}\end{array}$} & & E-bike HA & E-bike LA & $C B$ & Walking & \\
\hline & Mean absolute $\mathrm{VO}_{2 \text { peak }}$ & $1.50(.038)$ & $1.79(0.46)$ & $2.00(0.44)$ & $1.6(0.34)$ & $\begin{array}{l}<0.001 \text { overall, }<.05 \text {, all comparisons except HA vs. } \\
\text { Walk }(>.05)\end{array}$ \\
\hline & Percentage $\mathrm{VO}_{2 \text { peak }}$ & $54.9(11)$ & $65.7(8.1)$ & $72.8(6.4)$ & $59(9.1)$ & $\begin{array}{l}<0.001 \text { overall, <.05, all comparisons except HA vs. } \\
\text { Walk }(>.05)\end{array}$ \\
\hline & Mean estimated METs & $6.1(1.4)$ & $7.3(1.0)$ & $8.2(1.3)$ & $6.5(0.8)$ & $\begin{array}{l}<0.001 \text { overall, <.05, all comparisons except HA vs. } \\
\text { Walk }(>.05)\end{array}$ \\
\hline & Mean HR & $138.4(18)$ & $149(17.7)$ & $157.0(11.2)$ & $132.7(17.4)$ & $\begin{array}{l}<0.001 \text { overall, <.05, all comparisons except HA vs. } \\
\text { Walk }(>.05)\end{array}$ \\
\hline & Percentage HR max & $74.5(8.7)$ & $80.3(8.7)$ & $84.6(5.2)$ & $71.5(9.2)$ & $\begin{array}{l}<0.001 \text { overall, <.05, all comparisons except HA vs. } \\
\text { Walk }(>.05)\end{array}$ \\
\hline \multirow[t]{4}{*}{ Hansen, 2017 [21] } & & E-bike HA & E-Bike LA & $\mathrm{CB}$ & & \\
\hline & Mean absolute $\mathrm{VO}_{2}$ & $1.72(0.54)$ & $1.89(0.62)$ & $1.85(0.52)$ & & .02 overall, $.04 \mathrm{LA}$ vs. HA, > .05 CB vs. LA, CB vs. HA \\
\hline & Percentage $\mathrm{VO}_{2 \text { peak }}$ & $68(7.1)$ & $74(6.2)$ & $73(4.6)$ & & .01 overall, .03 LA vs. HA, > .05 CB vs. LA, CB vs. HA \\
\hline & Mean estimated METs & $6(1.8)$ & $6.6(2)$ & $6.4(1.6)$ & & .02 overall; .027 HA vs. LA; $>.05, C B$ vs $L A, C B$ vs. HA \\
\hline \multirow{2}{*}{$\begin{array}{l}\text { Hochsmann, } 2017 \\
\text { [30] }\end{array}$} & (Median, IQR) & E-bike & $C B$ & & & \\
\hline & Percentage HR max ${ }^{+}$ & $\begin{array}{l}74.9(67.4 \\
82.8)\end{array}$ & $\begin{array}{l}73.3(67.7 \\
78.2)\end{array}$ & & & NC \\
\hline \multirow{6}{*}{$\begin{array}{l}\text { Langford, } 2017 \\
{[23]^{\mathrm{a}, \mathrm{c}}}\end{array}$} & & E-bike & $C B$ & Walking & & \\
\hline & Mean relative $\mathrm{VO}_{2}$ & $16.95(5.17)$ & $19.32(5.47)$ & $15.12(5.35)$ & & NC \\
\hline & $\begin{array}{l}\text { Mean relative EE per } \\
\text { minute }\end{array}$ & $0.08(0.03)$ & $0.10(0.02)$ & $0.07(0.03)$ & & NC \\
\hline & Mean estimated METs & 5.1 & 5.8 & 4.5 & & NC \\
\hline & Mean HR & $\begin{array}{l}121.35 \\
(17.04)\end{array}$ & $\begin{array}{l}127.45 \\
(18.17)\end{array}$ & $\begin{array}{l}115.25 \\
(14.41)\end{array}$ & & NC \\
\hline & Mean power output & $63.28(22.89)$ & $73.13(35.79)$ & NA & & NC \\
\hline \multirow[t]{7}{*}{ 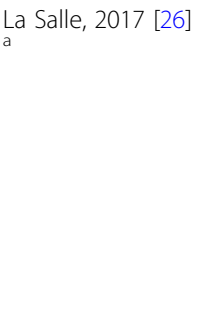 } & & E-bike & $C B$ & & & \\
\hline & Mean absolute $\mathrm{VO}_{2}$ & $2.3(0.1)$ & $2.5(0.1)$ & & & .45 \\
\hline & Percentage $\mathrm{VO}_{2 \max }$ & $66.4(2.6)$ & $68(2.8)$ & & & NR \\
\hline & Mean estimated METs & $8.3(0.5)$ & $8.5(0.6)$ & & & .65 \\
\hline & Mean HR & $147(5)$ & $149(5)$ & & & .064 \\
\hline & Percentage HR max & $79.1(2.4)$ & $80.4(2.6)$ & & & NR \\
\hline & Mean power output & $115(11)$ & $128(11)$ & & & .38 \\
\hline \multirow[t]{4}{*}{ Louis, 2012 [27] ${ }^{\mathrm{b}}$} & Trained & E-bike HA & E-bike LA & E-bike NA & & \\
\hline & Mean relative $\mathrm{VO}_{2}$ & $14.7(2.0)$ & $19.5(2.4)$ & $22.9(2.2)$ & & $<.05$, all comparisons \\
\hline & Mean estimated METs & $4.2(0.6)$ & $5.6(0.7)$ & $6.5(0.6)$ & & $<.05$, all comparisons \\
\hline & Mean absolute EE per & $5.1(0.8)$ & $7.6(0.8)$ & $7.8(0.5)$ & & $<.05$, all comparisons \\
\hline
\end{tabular}


Table 2 Physical activity intensity outcomes of interest measured during rides ${ }^{*}$ (Continued)

\begin{tabular}{|c|c|c|c|c|c|c|}
\hline \multirow[t]{2}{*}{ Study } & \multirow[t]{2}{*}{ Outcomes } & \multicolumn{5}{|c|}{ Results; mean (SD) } \\
\hline & & E-bike & $\begin{array}{l}\text { Comparison } \\
1\end{array}$ & $\begin{array}{l}\text { Comparison } \\
2\end{array}$ & $\begin{array}{l}\text { Comparison } \\
3\end{array}$ & Significance testing, $p$ value \\
\hline & Mean HR & $77.7(11)$ & $89.4(10.2)$ & $92.8(11.6)$ & & $<.05$, all comparisons \\
\hline & Mean power output & $47.3(9.1)$ & $83.6(4.0)$ & $104.2(4.2)$ & & $<.05$, all comparisons \\
\hline & Untrained & E-bike HA & E-bike LA & E-bike NA & & \\
\hline & Mean relative $\mathrm{VO}_{2}$ & $15.0(2.0)$ & $21.7(4.2)$ & $23.4(3.6)$ & & $<.05$, all comparisons \\
\hline & Mean estimated METs & $4.3(0.6)$ & $6.2(1.2)$ & $6.7(1.0)$ & & $<.05$, all comparisons \\
\hline & $\begin{array}{l}\text { Mean absolute EE per } \\
\text { minute }\end{array}$ & $4.9(0.8)$ & $6.7(0.8)$ & $7.5(0.9)$ & & $<.05$, all comparisons \\
\hline & Mean HR & $96.8(16.8)$ & $116.8(21.7)$ & $116.7(16.2)$ & & $<.05$, all comparisons \\
\hline & Mean power output & $40.0(7.1)$ & $79.8(4.8)$ & $99.9(6.9)$ & & $<.05$, all comparisons \\
\hline \multirow[t]{2}{*}{ Meyer 2014 [28] ${ }^{a}$} & & E-bike & E-bike NA & & & \\
\hline & Mean HR & 94.71 & 131.31 & & & NC \\
\hline \multirow{4}{*}{$\begin{array}{l}\text { Peterman, } 2016 \\
\text { [13] }\end{array}$} & & E-bike & & & & \\
\hline & Mean estimate METs & $4.9(1.2$ & & & & \\
\hline & $\begin{array}{l}\text { Mean absolute EE per } \\
\text { minute }\end{array}$ & $6.5(1.9)$ & & & & \\
\hline & Percentage HR max & $72.1(5.4)$ & & & & \\
\hline \multirow[t]{5}{*}{ Simons, 2009 [20] } & & E-bike HA & E-bike LA & E-bike NA & & \\
\hline & Mean estimated METs & $5.2(1.4)$ & $5.7(1.2)$ & $6.1(1.6)$ & & $<.05 \mathrm{HA}$ and NA, >.05 HA vs. LA, LA vs. NA \\
\hline & Mean HR & $112.4(22.9)$ & $116.2(22.4)$ & $123.8(23.2)$ & & $<.05$ NA vs. HA; NA vs. LA, >.05 HA vs. LA \\
\hline & Percentage HR max & $67.1(14.1)$ & $69.3(13.5)$ & $73.9(14.5)$ & & $<.05$ NA vs. HA; NA vs. LA, >.05 HA vs. LA \\
\hline & Mean absolute power & $94.2(29.2)$ & $101.8(24.8)$ & $118.2(30.9)$ & & $<.05$ All comparisons \\
\hline \multirow[t]{6}{*}{ Sperlich, 2012 [19] } & & E-bike & $C B$ & & & \\
\hline & Mean relative $\mathrm{VO}_{2}$ & $18(3.8)$ & $25.5(4.8)$ & & & $<.05, \mathrm{ES}=1.73$ \\
\hline & Mean absolute $\mathrm{VO}_{2}$ & $1.33(0.35)$ & $1.77(0.43)$ & & & $<.05, \mathrm{ES}=1.12$ \\
\hline & Mean estimated METs & $5.2(1.7)$ & $7.1(1.4)$ & & & $<.05, \mathrm{ES}=1.22$ \\
\hline & Mean HR & $105(20)$ & $133(19)$ & & & $<.05, \mathrm{ES}=1.53$ \\
\hline & Mean absolute power & $363(23)$ & $415(28)$ & & & $<.05, E S=2.02$ \\
\hline \multirow[t]{3}{*}{ Theurel, 2011 [24] } & & E-bike & $C B$ & & & \\
\hline & $\begin{array}{l}\text { Mean absolute EE per } \\
\text { minute }\end{array}$ & $5.6(1.3)$ & $5.9(1.8)$ & & & NR \\
\hline & Mean HR & NR & NR & & & $.02,3 \%$ lower with e-bike \\
\hline \multirow[t]{3}{*}{ Theurel, 2012 [25] } & & E-bike & CB & & & \\
\hline & Mean relative $\mathrm{VO}_{2}$ & $29(5)$ & $37(5)$ & & & $<.001$ \\
\hline & Mean HR & $136(23)$ & 167 (17) & & & $<.001$ \\
\hline
\end{tabular}

"Given the difference in the cycle routes conducted mean values or percentage of maximum for outcomes related to physical activity intensity are reported (e.g., Mean $\mathrm{VO}_{2 \text { peak }}$ mean heart rate, mean energy expenditure). For additional physical activity related outcomes reported in the studies see Additional file 4

${ }^{+}$reported for only a subsample of the group ( $n=5$ e-bikes, $n=4$ conventional bike)

$E E$ energy expenditure, $H R$ heart rate, $M E T s$ metabolic equivalent, $V_{2}$ volume of oxygen, $V_{2}$ oxygen intake value; $V_{2}$ max highest oxygen intake value attainable for an individual, $V O_{2 \text { peak }}$ the highest oxygen intake value obtained on a specific test, $C B$ conventional bike, $H A$ high assistance, $L A$ low assistance, $N A$ no assistance

ES effect size measured as Cohen's d, NC not conducted, $N R$ not reported

Relative $\mathrm{VO}_{2}, \mathrm{VO}_{2 \max }$ and $\mathrm{VO}_{2 \text { peak }}$ measured as $\mathrm{ml} / \mathrm{min} / \mathrm{kg} ;$ Absolute $\mathrm{VO}_{2}, \mathrm{VO}_{2 \max }$ and $\mathrm{VO}_{2 \text { peak }}$ measured in I/min; Mean absolute energy expenditure measured in $\mathrm{kcal} / \mathrm{min}$; Mean relative energy expenditure measured in $\mathrm{kcal} / \mathrm{kg} / \mathrm{min}$; Mean heart rate measured in beats per minute (bpm); Mean power output measured in Watts, Estimated METs measured using assumption that resting energy expenditure (i.e.,1 MET) $=3.5 \mathrm{ml} / \mathrm{kg} / \mathrm{min} ; \mathrm{Measured}$ METs measured through assessed individual resting energy expenditure

${ }^{a}$ Results are reported to total cycle routes. Studies separated results for different route topography. See Additional file 3 for details on different cycling topography; ${ }^{\text {b }}$ Participants completed same activity at three different speeds, self-selected speed reported; ${ }^{c}$ Total sample analyses not conducted, see Additional file 3 for analyses between ride segments 
assistance. Heart rate showed a trend towards being lower while walking compared to e-cycling [18, 23, 32].

\section{Power output}

Five studies assessed power output during conditions [19, 20, 23, 26, 27]. Mean power output was lower while riding an e-bike compared to a conventional bike or e-cycling with no assistance. Riding an e-bike on high assistance compared to low assistance led to significantly lower power outputs.

Overall, e-cycling was performed at a moderate intensity, but the intensity was lower than during conventional cycling. Most studies reported significant differences in the associated outcomes between e-cycling and conventional cycling. However, one study found no differences in physiological markers of intensity between e-cycling and conventional cycling [26]. While the evidence is limited, e-cycling appears to be performed at a greater intensity than walking.

\section{Impact of topography}

Five studies directly compared the impact of e-cycling in varying topographies (Additional file 3). The energy cost during e-cycling and conventional cycling uphill ranged from 5.2 to 6.8 and 7.2 to 8.5 METs respectively. This difference was statistically significant in the three studies that conducted hypothesis testing. Examination of means and medians suggested that energy expenditure (METs) during downhill and flat sections were lower while e-cycling compared to conventional cycling, but that this difference in energy cost was less distinct than during uphill sections. Across all studies, greater elevation gains in routes led to higher energy cost for both e-cycling and conventional cycling compared to flat routes or those conducted indoors. Differences in heart rate between e-cycling and conventional cycling appear to be greater during uphill sections, except for one study [19] that reported similar differences in heart rate between cycling conditions across all topographies.

\section{Physical fitness}

A pilot randomized control trial of physically inactive individuals reported an increase in peak oxygen uptake $\left(\mathrm{VO}_{2 \text { peak }}\right)$ of $10 \%$ following 4-weeks of e-cycling compared to a $6 \%$ increase following 4-weeks of conventional cycling [30] (Table 3). In a similar population, using a single-group quasi-experimental design, one study reported an $8 \%$ increase in $\mathrm{VO}_{2 \text { peak }}$ following 4-weeks of e-cyling [13] and another reported a 7.7\% increase in $\mathrm{VO}_{2 \text { peak }}$ following 3-months of e-cycling [31]. When separated into low and high fitness groups a significant increase in $\mathrm{VO}_{2 \text { peak }}$ was reported only in individuals with low levels of fitness, with a $9.6 \%$ increase compared to a $1.5 \%$ increase in high fitness individuals [31]. Gender differences were reported in one study following 6-weeks of e-cycling with a 2 and $7 \%$ increase in $\mathrm{VO}_{2 \text { peak }}$ in physically inactive men and women respectively [29]. Gender differences were also reported in maximum power output with women reporting lower increases in maximum power than men following a 6-week and 5-month intervention [29, 32].

\section{Health outcomes}

Three studies examined the impact of e-cycling on health outcomes beyond fitness (Table 3), for which the outcomes assessed were heterogeneous. After 4-weeks of e-cycling there were no changes in systolic or diastolic blood pressure at rest $[13,30]$. There was no evidence of a difference in blood pressure whilst cycling between conventional cycling and e-cycling [30]. Peterman and colleagues [13] reported no changes in insulin resistance or lipid profiles following 4-weeks of e-cycling. However, a significant reduction in 2-h post plasma glucose concentration was reported. No changes were reported in the one study examining quality of life following 8 weeks of e-cycling [33].

\section{Quality assessment and quality of the evidence}

The global rating of acute studies yielded six moderate and five weak ratings according to the EPHPP tool (Table 4). Ten studies were rated as weak for representativeness of the target population, often due to a failure to report how participants were recruited. Methods of assessment were rated as strong. The repeated nature of conditions ensured the control of confounders, therefore yielding a strong rating. Overall there was moderate evidence that e-cycling could lead to physical activity at an intensity associated with beneficial health outcomes [14]. A global rating of strong was given to one longitudinal study, moderate was given to four studies and weak to one study. There was moderate evidence that e-cycling could lead to increased fitness. The evidence related to the impact of e-cycling on additional health outcomes was inconclusive.

\section{Discussion}

The aim of the current review was to assess the intensity of physical activity when riding an e-bike, and to examine the physiological and psychological outcomes associated with e-cycling. Where possible these outcomes were compared to traditional methods of active travel (i.e., walking and cycling). Eleven acute and six longitudinal studies were identified. There was moderate evidence that e-cycling provides moderate intensity physical activity in both physically active and inactive individuals. Furthermore, there was moderate evidence that e-cycling positively impacted cardiorespiratory fitness in physically inactive individuals. The impact of e-cycling on health outcomes beyond physical fitness was inconclusive given the sparsity of current research.

\section{Quality of the evidence}

The quality of all studies, bar one [30], was weak to moderate. These ratings should be viewed with caution 
Table 3 Results of longitudinal intervention studies

\begin{tabular}{|c|c|c|c|c|c|c|}
\hline \multirow[t]{3}{*}{ Study } & \multirow[t]{3}{*}{ Outcomes } & \multicolumn{5}{|c|}{ Results, mean, SD (95\% Cl) } \\
\hline & & \multicolumn{2}{|l|}{ Intervention } & \multicolumn{2}{|l|}{ Control } & \multirow[t]{2}{*}{ Significance, $p$-value } \\
\hline & & Pre & Post & Pre & Post & \\
\hline & & E-bike & & & & \\
\hline \multirow[t]{4}{*}{ Cooper, 2018 [32] } & Max absolute power & $157.5(55.7)$ & $174.3(70.8)$ & & & NC \\
\hline & Men & $182.1(51.5)$ & $206.2(64.9)$ & & & NC \\
\hline & Women & $118.9(38.9)$ & $124.3(49.0)$ & & & NC \\
\hline & & E-bike & & $\mathrm{NE}$ & & Within groups \\
\hline \multirow[t]{13}{*}{ De Geus, 2013 [29] } & Absolute $\mathrm{VO}_{2 \text { peak }}$ & & & & & \\
\hline & Men & $2.56(0.35)$ & $2.61(0.38)$ & $2.62(0.46)$ & $2.56(0.35)$ & $>$ >0.025 E-bike, NE \\
\hline & Women & $1.94(0.37)$ & $2.07(0.41)$ & $1.91(0.35)$ & $1.94(0.37)$ & >.0.025 E-bike, NE \\
\hline & Relative $\mathrm{VO}_{2 \text { peak }}$ & & & & & \\
\hline & Men & $30.2(4.3)$ & $30.7(5.6)$ & $30.8(4.9)$ & $30.2(4.3)$ & $>0.025$ E-bike, NE \\
\hline & Women & $30.0(6.0)$ & $32.3(6.5)$ & $29.4(5.1)$ & $30.0(6.0)$ & >.0.025 E-bike, NE \\
\hline & Absolute max power & & & & & \\
\hline & Men & $169.5(19.9)$ & $192.1(28.7)$ & $173.8(27.1)$ & $169.5(19.9)$ & $<.0 .025$ E-bike, $>.0 .025 \mathrm{NE}$ \\
\hline & Women & $130.9(21.6)$ & $145.9(24.8)$ & $131.1(21.7)$ & $130.9(21.6)$ & $<.0 .025$ E-bike, $>.0 .025 \mathrm{NE}$ \\
\hline & Relative max power & & & & & \\
\hline & Men & $2.00(0.28)$ & $2.30(0.40)$ & $2.05(0.35)$ & $2.00(0.28)$ & $<.0 .025$ E-bike, $>.0 .025 \mathrm{NE}$ \\
\hline & Women & $2.03(0.41)$ & $2.30(0.55)$ & $2.04(0.43)$ & $2.03(0.41)$ & $<.0 .025$ E-bike, $>.0 .025 \mathrm{NE}$ \\
\hline & & E-bike & & $C B$ & & \\
\hline \multirow[t]{9}{*}{ Hochsmann, 2017 [30] } & Relative $\mathrm{VO}_{\text {2peak }}$ & $35.7(5.8)$ & $39.3(8.3)$ & $36.4(7.3)$ & $38.6(6.2)$ & $0.327,1.4(-1.4-4.1)^{+}$ \\
\hline & Relative power output & $2.9(0.6)$ & $3.2(0.6)$ & $3(0.5)$ & $3.3(0.5)$ & $0.995,0.0(-0.1-0.1)^{+}$ \\
\hline & Resting HR & $64.7(6.5)$ & $65.1(7.6)$ & $68.8(8.8)$ & $65.5(10.6)$ & $0.505,2.0(-4.2-8.2)^{+}$ \\
\hline & HR at $100 \mathrm{~W}$ max text & $113.4(9.2)$ & $111.5(7.7)$ & $113.4(15.9)$ & $109.2(14.2)$ & $0.219,2.4(-1.5-6.2)^{+}$ \\
\hline & SBP at rest & $125.9(13.8)$ & $124.1(11.3)$ & $127.3(10.6)$ & $123.1(12.4)$ & $0.538,2.0(-4.5-8.5)^{+}$ \\
\hline & DBP at rest & $82.4(8.5)$ & $82.1(8.2)$ & $87.7(8)$ & $84.5(8.8)$ & $0.625,1.2(-3.9-6.3)^{+}$ \\
\hline & SBP@100W & $174.1(22.9)$ & $160.3(21.2)$ & $160.8(20)$ & $150.4(18.5)$ & $0.93,-0.4(-9.4-8.7)^{+}$ \\
\hline & DBP@ @ 100 W & $86.2(8.3)$ & $81.9(6.5)$ & $88(7.1)$ & $84(8.1)$ & $0.709,-1.1(-7.5-5.2)^{+}$ \\
\hline & & E-bike & & & & \\
\hline \multirow[t]{6}{*}{ Malnes, 2016 [31] } & Relative $\mathrm{VO}_{2 \text { peak }}$ & $34.1(31.6,36.7)$ & $36.5(34.4,38.6)$ & & & $<.001$ \\
\hline & Relative $\mathrm{VO}_{2 \text { peak, }} \%$ gain & & $7.7(4.3,11.1)$ & & & \\
\hline & High Fitness & & $1.5(-5.6,8.6)$ & & & 0.626 \\
\hline & Low Fitness & & $9.6(5.9,13.3)$ & & & $<.05$ \\
\hline & Peak HR & $181(175,187)$ & $180(174,186)$ & & & 0.429 \\
\hline & & E-bike commute & & Passive commute & & \\
\hline \multirow[t]{3}{*}{ Page, 2017 [33] } & QOL (baseline and week 8) & $38.00(3.86)$ & $39.67(4.47)$ & $29.63(6.57)$ & $35.71(5.59)$ & >.05 E-bike, Passive commute \\
\hline & OQL (week 4) & & $38.84(4.16)$ & & $32.67(6.08)$ & $<.01, E S=0.28$ \\
\hline & & E-bike & & & & \\
\hline \multirow[t]{6}{*}{ Peterman, 2016 [13] } & Absolute $\mathrm{VO}_{2 \max }$ & $2.21(0.48)$ & $2.39(0.52)$ & & & $<.05$ \\
\hline & MVPA & $28.1(17.5)$ & $29.0(20.2)$ & & & $>.05$ \\
\hline & MVPA10+ & $11.7(14.3)$ & $13.0(15.2)$ & & & $>.05$ \\
\hline & Absolute max power & $165.1(37.1)$ & $189.3(38.3)$ & & & $<.05$ \\
\hline & Fasting glucose & $4.99(0.52)$ & $5.02(0.47)$ & & & $>.05$ \\
\hline & $2 \mathrm{~h}$ post plasma glucose & $5.53(1.18)$ & $5.03(0.91)$ & & & $<.05$ \\
\hline
\end{tabular}


Table 3 Results of longitudinal intervention studies (Continued)

\begin{tabular}{|c|c|c|c|c|c|c|}
\hline \multirow[t]{3}{*}{ Study } & \multirow[t]{3}{*}{ Outcomes } & \multicolumn{5}{|c|}{ Results, mean, SD (95\% Cl) } \\
\hline & & \multicolumn{2}{|l|}{ Intervention } & \multicolumn{2}{|c|}{ Control } & \multirow[t]{2}{*}{ Significance, $p$-value } \\
\hline & & Pre & Post & Pre & Post & \\
\hline & HOMA & $2.46(0.95)$ & $2.55(0.82)$ & & & $>.05$ \\
\hline & Total cholesterol & $3.90(0.87)$ & $3.92(0.79)$ & & & $>.05$ \\
\hline & LDL & $2.33(0.8)$ & $2.34(0.71)$ & & & $>.05$ \\
\hline & $\mathrm{HDL}$ & $1.21(0.24)$ & $1.18(0.22)$ & & & $>.05$ \\
\hline & Triglycerides & $0.95(0.42)$ & $0.91(0.27)$ & & & $>.05$ \\
\hline & MAP & $84.6(10.5)$ & $83.2(9.4)$ & & & $>.05$ \\
\hline & SBP & $110.0(12.4)$ & $109.1(10.9)$ & & & $>.05$ \\
\hline & DBP & $67.7(8.8)$ & $67.0(8.0)$ & & & $>.05$ \\
\hline
\end{tabular}

${ }^{+}$difference between groups, 95\% Cl, ES = effect size

Distance (total and weekly) measured in kilometres; Duration (total and weekly) measured in minutes

$N E$ no activity, $C B$ conventional bike

$S B P$ systolic blood pressure, $D B P$ diastolic blood pressure, $M A P$ mean arterial blood pressure, $Q O L$ quality of life, $L D L$ low density lipo-protein, $H D L$ high density lipo-protein, HOMA measure of insulin sensitivity using homeostatic model assessment, MVPA moderate to vigorous physical activity, MVPA10+ moderate to vigorous physical activity of bout of 10-min or greater, $W$ watts

$\mathrm{VO}_{2 \max }=$ highest oxygen value attainable for an individual, $\mathrm{VO}_{2 \text { peak }}=$ the highest oxygen intake value obtained on a specific test

Relative $\mathrm{VO}_{2 \text { max }}$ and $\mathrm{VO}_{2 \text { peak }}$ measured as $\mathrm{ml} / \mathrm{min} / \mathrm{kg} ;$ Absolute $\mathrm{VO}_{2 \text { max }}$ and $\mathrm{VO}_{2 \text { peak }}$ measured in $\mathrm{I} / \mathrm{min}$ Mean energy expenditure measured in $\mathrm{kcal} / \mathrm{min} ;$ Mean heart rate or peak heart rate measured in beats per minute (bpm); Mean absolute max power measured in Watts, Mean relative power measured in watts/kg; glucose, cholesterol, LDL, HDL, Triglycerides measured in $\mathrm{mmol} / \mathrm{L}$; blood pressure measured in millimeter of mercury (mmHg), MVPA and MVPA10+ measured in minutes per day

Table 4 Quality assessment of included studies according to the Effective Public Health Practice Project tool

\begin{tabular}{|c|c|c|c|c|c|c|c|}
\hline \multirow[t]{2}{*}{ Study } & \multicolumn{6}{|c|}{ Component rating } & \multirow{2}{*}{$\begin{array}{l}\text { Global } \\
\text { rating }\end{array}$} \\
\hline & Selection Bias & Design & Confounders & Blinding & Methods & Drop-outs & \\
\hline \multicolumn{8}{|l|}{ Acute studies } \\
\hline Bernsten [22] & Weak & Strong & Strong & Weak & Strong & Strong & Moderate \\
\hline Gojanovic [18] & Weak & Moderate & Strong & Weak & Strong & Strong & Moderate \\
\hline Hansen [21] & Moderate & Strong & Strong & Weak & Strong & Strong & Moderate \\
\hline Langford [23] & Weak & Moderate & Strong & Weak & Strong & Moderate & Moderate \\
\hline La Salle [26] & Weak & Strong & Strong & Weak & Strong & Strong & Moderate \\
\hline Louis [27] & Weak & Strong & Strong & Weak & Strong & Weak & Weak \\
\hline Meyer [28] & Weak & Weak & Strong & Weak & Strong & Weak & Weak \\
\hline Simons [20] & Weak & Moderate & Strong & Weak & Strong & Strong & Moderate \\
\hline Sperlich & Weak & Strong & Strong & Weak & Strong & Weak & Weak \\
\hline Theurel, 2011 [24] & Weak & Weak & Strong & Weak & Strong & Weak & Weak \\
\hline Theurel, 2012 [25] & Weak & Weak & Strong & Weak & Strong & Weak & Weak \\
\hline \multicolumn{8}{|l|}{ Longitudinal studies } \\
\hline Cooper [32] & Moderate & Moderate & Strong & Weak & Strong & Moderate & Moderate \\
\hline De Geus [29] & Weak & Moderate & Strong & Weak & Strong & Moderate & Moderate \\
\hline Hochsmann [30] & Moderate & Strong & Strong & Weak & Strong & Strong & Strong \\
\hline Malnes [31] & Weak & Moderate & Strong & Weak & Strong & Strong & Moderate \\
\hline Page [33] & Moderate & Weak & Weak & Weak & Strong & Weak & Weak \\
\hline Peterman [13] & Weak & Moderate & Strong & Weak & Strong & Moderate & Moderate \\
\hline
\end{tabular}

${ }^{\mathrm{a}}$ Strong = no weak component rating; moderate = one weak component rating; weak = two or more weak component ratings Note: blinding was not included in the overall global rating calculation 
as the purpose of physiological studies, such as the acute experiments reported here, is to explore a specific event in a controlled environment with less focus on obtaining representative samples. As such, many studies did not report how participants were recruited, leading to a weak rating for the selection bias component of the assessment. Study design, control of confounders and methods of assessment are often considered more crucial in these designs, all of which were strong in the acute studies reported here. Furthermore, while blinding is often unachievable in physical activity interventions, the use of objective methodology limits the impact of research bias on the outcomes.

Regarding longitudinal studies, methods of data collection were consistently strong, but with large variation in representativeness, design and reporting of withdrawals and dropouts. Confounders were considered in the context of differences between groups and were therefore rated as strong if studies used a single-group design. One pilot randomized control trial was conducted and was rated as strong [30]. Overall, there was a lack of high-quality longitudinal intervention-based research including pre-post measures examining the impact of e-cycling on physiological and psychological health outcomes.

\section{The impact of e-cycling on physical activity intensity}

To accrue health benefits, The American College of Sports Medicine recommend healthy adults engage in moderate-to-vigorous physical activity for 150-min per week [14]. Moderate intensity activity is classified as three to six metabolic equivalents (METs) and vigorous intensity activity at six METs or above. The current review suggests that e-cycling, even while using a high assistance mode, provides physical activity of at least moderate intensity on a variety of terrain, including downhill. Furthermore, e-cycling can elicit vigorous activity during uphill riding [18] and during rides with highly varied terrain $[18,26]$. Interestingly, Bernsten and colleagues [22] reported that mean estimated METs were lower than mean measured METs during e-cycling. Estimated METs have been suggested to overestimate resting energy expenditure, thereby underestimating activity energy expenditure [34]. As such, the mean estimated METs reported in this review provide a conservative estimate of exercise intensity.

Relative physiological outcomes further suggest that e-cycling is performed at a moderate intensity with the percent of maximum heart rate ranging from 67.1 to 79.1 and the percent of $\mathrm{VO}_{2 \text { peak/max }}$ ranging from 51 to 75. These values exceed the hypothesised minimum intensity thresholds required for improvements in cardiorespiratory fitness in healthy adults $[14,35,36]$.

\section{E-cycling vs. traditional active transportation}

Three studies compared e-cycling to walking [18, 23, 32] of which one compared the two modes on the same route [23]. In this study walking led to lower oxygen uptake than e-cycling across all topographies, though significant MET differences were only reported during uphill sections, with e-cycling expending more energy than walking. The few studies conducted suggest e-cycling is performed at a higher intensity than walking, however, more studies are needed to confirm these trends.

In relation to conventional cycling, this review suggests that e-cycling elicits lower physiological markers of intensity than conventional cycling, however the strength of this finding depends on the physiological assessment measure and route topography. Overall, mean percent of $\mathrm{VO}_{2 \max /}$ peak is similar between conventional cycling and e-cycling ranging from 58 to $74 \%$ and 51 to $73 \%$ respectively. Studies examining active commuting on conventional bikes have reported similar mean percent of $\mathrm{VO}_{2 \max }$ in healthy adults ranging from 57 to $79 \%[6,37]$. However, mean relative oxygen uptake is lower during e-cycling compared to conventional cycling or e-cycling without assistance. Similarly, means and medians of estimated METs are consistently higher during conventional cycling or e-cycling without assistance compared to assisted e-cycling, with values ranging from 6.1 to 8.5 and 4.9 to 8.3 respectively, though the significance of the differences varied across studies.

La Salle and colleagues [26] reported similar MET values between e-cycling and conventional cycling. However, the values reported were substantially higher than those reported in other studies, with mean estimated METs of 8.3 and 8.5 for e-cycling and conventional cycling respectively. Participant demographics may have accounted for these differences, since participants were younger and had previous cycling experience. These participants may have had higher aerobic capacity and therefore self-selected a higher intensity activity level at which to complete the conditions. This is likely given that the relative intensity of activity is similar in studies of e-cycling in physically inactive individuals $[13,18-20$, $30,32]$. When given the choice to self-select pace and intensity individuals may select a similar physiological intensity across activities regardless of the mechanical assistance, thereby resulting in similar physiological outcomes. In support of this, when individuals were required to maintain a cycling cadence of 60 revolutions per minute throughout a condition, there were significant differences in oxygen uptake and heart rate between e-bikes and conventional bikes [18] compared to studies in which individuals were able to self-selected their intensity [21, 22, 26]. Similarly, when instructed to complete 60-meters of riding in 10 -sec for a total of 30 -min the reported relative $\mathrm{VO}_{2 \max }$ was $29 \mathrm{ml} / \mathrm{min} / \mathrm{kg}$ for e-cycling and $37 \mathrm{ml} / \mathrm{min} / \mathrm{kg}$ for conventional cycling [25]. This suggests that performing the same amount of work requires more effort on a conventional bike than an e-bike, but that human beings reduce the amount of work conducted on a conventional bike, through choosing a 
slower speed, to account for the increase in expended effort.

In hilly terrain, where there is less opportunity to adjust effort levels to produce comparable intensity levels, the differences between conventional cycling and e-cycling may become more pronounced, with e-cycling requiring lower intensity activity, as found in studies comprised of routes with hilly features $[18,23]$. This suggests that e-bikes are less sensitive to environmental factors such as topography. Therefore, physiological measures of intensity are lower on the e-bike than those reported on a conventional bike during uphill riding. The reduced intensity required during uphill riding when using an e-bike is one of the leading arguments for the promotion of e-bikes as an alternative mode of active transportation.

\section{E-cycling and health}

In the current review three studies provided weekly e-cycling goals for physically inactive individuals in the context of active commuting [13, 29, 30]. Two of these studies reported increases in $\mathrm{VO}_{2 \text { peak }}$ and maximum power output following 4-weeks of e-cycling [13, 30]. In contrast de Geus and colleagues [10] reported no changes in $\mathrm{VO}_{2 \text { peak }}$ following a 6-week intervention, though differences in maximum power output were seen. Differences between studies could be due to distance cycled. Specifically, both Hochsmann [30] and Peterman and colleagues [13] reported cycling distances of $70 \mathrm{~km}$ and $69.4 \mathrm{~km}$ per week respectively, compared to $54.3 \mathrm{~km}$ per week reported by de Geus [10]. The two studies reporting significant increases in fitness also described self-selected riding intensities of between 72.1 and $74.9 \%$ of maximum heart rate (within the moderate intensity zone $[13,30]$ with an average of $205 \mathrm{~min}( \pm 43.3)$ of e-cycling per week [13]. This suggests that e-cycling can contribute to meeting weekly physical activity guidelines.

Without the provision of e-cycling goals, single group studies with physically inactive individuals reported increases in maximal power output of 7 to $10 \%$ over $3-8$ months, despite lower average distance travelled than other studies [31,32]. Fitness benefits were greatest in individuals classified as having low fitness [31], similar to findings with conventional cycling [6]. These results suggest that in the absence of specific goals (i.e., under free living conditions), participants engage in e-cycling and this e-cycling can contribute to improvements in fitness.

Beyond cardiorespiratory fitness, there is a lack of research examining the impact of e-cycling on physiological or psychological health outcomes, limiting our ability to draw conclusions. Peterman and colleagues [13] reported a decrease in 2-h plasma glucose during an oral glucose tolerance test after 4-weeks of e-cycling. This finding is in line with studies that have examined the impact of exercise on 2-h post exercise glucose concentrations in obese individuals $[38,39]$ but is novel in the context of e-cycling and conventional cycling. In the same study, no other metabolic changes were reported. Similar null effects on metabolic outcomes were reported in two systematic reviews on conventional cycling $[37,40]$.

\section{E-cycling for public health?}

Overall e-cycling can elicit at least moderate intensity physical activity. However, total energy expenditure when riding an e-bike is lower than when riding a conventional bike or walking over the same distance, given the reduced amount of time taken to complete a ride on an e-bike. Consequently, if e-cycling were to replace journeys made by walking or conventional cycling, individuals would have to ride for longer for comparable weekly energy expenditure. However, e-cycling is associated with lower ratings of perceived exertion than conventional cycling $[23,26]$, potentially enabling people to ride more frequently or for a longer duration. This possibility is supported by Hendriksen and colleagues [41], who reported that individuals in the Netherlands commuted $50 \%$ further with an e-bike than on a conventional bike.

Findings reported here suggest that e-cycling may be suitable for individuals with compromised health. Hansen and colleagues [21] showed that e-cycling elicited moderate intensity activity in older, obese individuals recovering from surgery due to coronary artery disease, while Cooper and colleagues [32] reported that e-cycling was feasible for middle-aged, overweight individuals with type 2 diabetes mellitus.

Overall, while there is a trend towards increased fitness following engagement in e-cycling interventions, more intervention research of a longer duration is required before the long-term impact of e-cycling on health can be determined. Fifty percent of the longitudinal studies in this review were approximately 1 -month in length. This may not be enough time to see changes in body composition and some metabolic outcomes. Longer trials with larger samples sizes should be conducted with a focus on including a range of health outcomes in addition to cardiorespiratory fitness. These trials should utilize randomized controlled designs and clearly report their target population, recruitment process and dropouts and/or withdrawals. Interventions should also be conducted in clinical populations where physical activity is compromised. In addition, more research is needed to understand the impact of e-cycling on health based on sex or fitness level.

It is also important to consider the negative outcomes associated with e-cycling when assessing their potential utilization for health promotion. In the USA, e-bike users reported feeling safer riding their e-bike than a conventional bike, stating that the e-bike helped them to avoid crashes due to their stability, powerful brakes and the acceleration to avoid incidents and keep up with traffic. However, riders reported cycling faster on an e-bike than a conventional bike and felt that other road users misjudged their speed leading to potentially dangerous situations [42]. In the Netherlands data suggest that, 
after controlling for age, gender and amount of cycling, use of an e-bike was associated with an increased risk of being involved in a crash compared to conventional cycling [43]. The severity of these crashes was not significantly different from conventional cycling [43]. More context specific research is required to enable a risk-benefit assessment of engaging specifically in e-cycling. Nevertheless, e-cyclists would be well advised to be appropriately trained and use safety equipment to minimize risk.

\section{Strengths and limitations}

This is the first review to examine the physical activity intensity, cardiorespiratory, metabolic and psychological outcomes associated with e-cycling. This review used two pragmatic tools to assess the quality of studies and to provide an overall rating of the evidence. These tools provided an overall representation of the strength of research evidence related to e-cycling and health. Limitations of this review include the fact that some published studies may not have been identified. However, our systematic and broad search strategy makes this unlikely. It is more likely that we did not identify eligible unpublished studies or those published in an alternative language to English. Sample sizes used in studies were small and sample size calculations were rarely reported. Therefore, caution should be taken when interpreting the statistical significance of evidence. Given the heterogeneity in outcome measurement we were unable to quantify the effects of e-cycling on outcomes of interest using meta-analyses. In addition, focus on quality of life as a psychological outcome may have meant studies examining psychological outcomes such as depression or anxiety were excluded.

\section{Conclusion}

The composite results of the 17 studies included in this novel systematic review provide moderate evidence that e-cycling elicits activity at an intensity high enough to promote some positive health outcomes. E-cycling leads to reduced activity volume and intensity over the same distance compared to conventional cycling. Therefore, e-cycling requires more frequent and longer rides to accrue comparable health benefits. However, given that most individuals travel by car to work [44] e-cycling offers a physically active alternative to the largely sedentary behaviour associated with motorized commuting. Furthermore, longitudinal studies suggest, with moderate confidence, that e-cycling can lead to increases in cardiorespiratory fitness. Longer and higher-quality intervention studies, with transparent reporting, are needed to develop a strong evidence-based understanding of the impact of e-cycling on cardiorespiratory health and to explore the impact of e-cycling on metabolic and psychological outcomes. This will extend the current body of knowledge and provide guidance on public health initiatives to promote e-cycling to improve population health.

\section{Endnote}

${ }^{1}$ The MET is an expression of energy cost and is calculated from rest where $1 \mathrm{MET}$ is estimated to equal $3.5 \mathrm{ml} / \mathrm{kg} / \mathrm{min}$

\section{Additional files}

Additional file 1: Example search strategy. (DOCX $12 \mathrm{~kb}$ )

Additional file 2: Description of overall strength of evidence criteria. (DOCX $12 \mathrm{~kb}$ )

Additional file 3: Outcomes of interest by route topography for acute experimental and quasi-experimental studies. (DOCX $23 \mathrm{~kb}$ )

Additional file 4: Additional physical activity outcomes measured in acute studies. (DOCX $28 \mathrm{~kb})$

Additional file 5: Additional physical activity outcomes measured in longitudinal studies. (DOCX $22 \mathrm{~kb}$ )

\section{Acknowledgements}

The authors would like to thank Sarah Koch and Kylie Gobereau for their help in translating study manuscripts.

\section{Funding}

This review was supported by the NIHR Bristol Nutrition Biomedical Research Centre.

\section{Availability of data and materials} Not applicable.

\section{Authors' contributions}

JEB conceptualized the review. JEB, RP, ARC and AP contributed to design and search strategy. JEB, SST, ARC and RP contribute to screening, data extraction and quality assessment. JEB drafted the full manuscript. All authors read and approved the final manuscript.

Ethics approval and consent to participate

Not applicable.

Consent for publication

Not applicable.

Competing interests

The authors declare that they have no competing interests.

\section{Publisher's Note}

Springer Nature remains neutral with regard to jurisdictional claims in published maps and institutional affiliations.

Received: 8 August 2018 Accepted: 8 November 2018 Published online: 21 November 2018

\section{References}

1. World Health Organization. Physical activity fact sheet; 2018. Available from: http://www.who.int/news-room/fact-sheets/detail/physical-activity.

2. Falconer $\mathrm{CL}$, Cooper AR, Flint E. Patterns and correlates of active commuting in adults with type 2 diabetes: cross-sectional evidence from UK Biobank. BMJ Open. 2017;7:10.

3. Laverty AA, Mindell JS, Webb EA, Millett C. Active travel to work and cardiovascular risk factors in the United Kingdom. Am J Prev Med. 2013; 45(3):282-8.

4. Celis-Morales CA, Lyall DM, Welsh P, Anderson J, Steell L, Guo Y, et al. Association between active commuting and incident cardiovascular disease, cancer, and mortality: prospective cohort study. BMJ. 2017;357:j1456. 
5. Kelly P, Kahlmeier S, Gotschi T, Orsini N, Richards J, Roberts N, et al. Systematic review and meta-analysis of reduction in all-cause mortality from walking and cycling and shape of dose response relationship. Int J Behav Nutr Phys Act. 2014;11:132.

6. Oja P, Titze S, Bauman A, de Geus B, Krenn P, Reger-Nash B, et al. Health benefits of cycling: a systematic review. Scand J Med Sci Sports. 2011;21(4):496-509.

7. Department for Transport. Road Use Statistics: Great Britain 20162016 [cited 2018 5th March]. Available from: https:/assets.publishing.senvice.gov.uk/government/ uploads/system/uploads/attachment_data/file/514912/road-use-statistics.pdf.

8. National Institute for Health and Care Excellence. Physical activity: walking and cycling 2012 [Available from: https://www.nice.org.uk/guidance/ph41/ resources/physical-activity-walking-and-cycling-pdf-1996352901061.

9. Department for Transport. Walking and Cycling Statistics, England: 2016: Statistical Release 2018 [Available from: https://assets.publishing.service.gov. uk/government/uploads/system/uploads/attachment_data/file/674503/ walking-and-cycling-statistics-england-2016.pdf.

10. De Geus B, Hendriksen I. Cycling for transport, physical activity and health: What about Pedelecs? 2015.

11. Fishman $\mathrm{E}$, Cherry $\mathrm{C}$. E-bikes in the mainstream: reviewing a decade of research. Transp Rev. 2016;36(1):72-91.

12. MacArthur J, Dill J, Person M. Electric bikes in North America. Transp Res Rec: J Transp Res Board. 2014;2468:123-30.

13. Peterman JE, Morris KL, Kram R, Byrnes WC. Pedelecs as a physically active transportation mode. Eur J Appl Physiol. 2016;116(8):1565-73.

14. Haskell WL, Lee I-M, Pate RR, Powell KE, Blair SN, Franklin BA, et al. Physical activity and public health: updated recommendation for adults from the American College of Sports Medicine and the American Heart Association. Med Sci Sports Exerc. 2007;39(8):1423-34.

15. Moher D, Liberati A, Tetzlaff J, Altman DG, Altman D, Antes G, et al. Preferred reporting items for systematic reviews and meta-analyses: the PRISMA statement. PLoS Med. 2009;6:e1000097.

16. Tools NCCFMa. Dictionary for the effective public health practice project quality assessment tool for quantitative studies. 2008 [Available from: https://merst.ca/ephpp/.

17. De Bourdeaudhuij I, Van Cauwenberghe E, Spittaels H, Oppert JM, Rostami C, Brug J, et al. School-based interventions promoting both physical activity and healthy eating in Europe: a systematic review within the HOPE project. Obes Rev. 2011:12(3):205-16.

18. Gojanovic B, Welker J, Iglesias K, Daucourt C, Gremion G. Electric bicycles as a new active transportation modality to promote health. Med Sci Sports Exerc. 2011;43(11):2204-10.

19. Sperlich B, Zinner C, Hebert-Losier K, Born DP, Holmberg HC. Biomechanical, cardiorespiratory, metabolic and perceived responses to electrically assisted cycling. Eur J Appl Physiol. 2012;112(12):4015-25

20. Simons M, Van Es E, Hendriksen I. Electrically assisted cycling: a new mode for meeting physical activity guidelines? Med Sci Sports Exerc. 2009;41(11):2097-102.

21. Hansen D, Soors A, Deluyker V, Frederix I, Dendale P. Electrical support during outdoor cycling in patients with coronary artery disease: impact on exercise intensity, volume and perception of effort. Acta Cardiol. 2017:73:1-8.

22. Berntsen S, Malnes L, Langaker A, Bere E. Physical activity when riding an electric assisted bicycle. Int J Behav Nutr Phys Act. 2017;14(1):55.

23. Langford BC, Cherry CR, Bassett DR, Fitzhugh EC, Dhakal N. Comparing physical activity of pedal-assist electric bikes with walking and conventional bicycles. J Transp Health. 2017;6:463-73.

24. Theurel J, Lepers R. Réponses cardiovasculaires lors de la distribution du courrier postal avec un vélo à assistance électrique. Mov Sport Sci. 2011/2;73:33-37.

25. Theurel J, Theurel A, Lepers R. Physiological and cognitive responses when riding an electrically assisted bicycle versus a classical bicycle. Ergonomics. 2012;55(7):773-81.

26. La Salle DT, Shute R, Heesch M, Slivka D. Demands of simulated commuting using an electrically assisted bicycle. Int J Exerc Sci. 2017;10(3):454-64.

27. Louis J, Brisswalter J, Morio C, Barla C, Temprado JJ. The electrically assisted bicycle: an alternative way to promote physical activity. Am J Phys Med Rehabil. 2012;91(11):931-40.

28. Meyer D, Steffan M, Senner V. Impact of electrical assistance on physiological parameters during cycling. Procedia Eng. 2014;72:150-5.

29. de Geus B, Kempenaers F, Lataire P, Meeusen R. Influence of electrically assisted cycling on physiological parameters in untrained subjects. Eur J Sport Sci. 2013;13(3):290-4

30. Höchsmann C, Meister S, Gehrig D, Gordon E, Li Y, Nussbaumer M et al. Effect of E-Bike Versus Bike Commuting on Cardiorespiratory
Fitness in Overweight Adults: A 4-Week Randomized Pilot Study. Clinical Journal of Sport Medicine. 2017:Publish Ahead of Print

31. Malnes L, Lobben S, Bere E, Tjelta LI, Kristoffersen M, Mildestvedt T, et al. How access to an E-bike affects bicycle use and cardiopulmonary fitness in inactive Norwegian adults: A pilot study. [Master's Thesis]. In press 2016.

32. Cooper A, Tibbitts B, England C, Procter D, Searle A, Sebire S, et al. The potential of electric bicycles to improve the health of people with type 2 diabetes: a feasibility study. Diabet Med. 2018.

33. Page NC, Nilsson VO. Active commuting: workplace health promotion for improved employee well-being and organizational behavior. Front Psychol. 2017;7:1994.

34. Byrne NM, Hills AP, Hunter GR, Weinsier RL, Schutz Y. Metabolic equivalent: one size does not fit all. J Appl Physiol. 2005;99(3):1112-9.

35. Swain DP, Franklin BA. VO2 reserve and the minimal intensity for improving cardiorespiratory fitness. Med Sci Sports Exerc. 2002:34(1):152-7.

36. Garber CE, Blissmer B, Deschenes MR, Franklin BA, Lamonte MJ, Lee I-M, et al. Quantity and quality of exercise for developing and maintaining cardiorespiratory, musculoskeletal, and Neuromotor fitness in apparently healthy adults: guidance for prescribing exercise. Med Sci Sports Exerc. 2011;43(7):1334-59.

37. De Geus B, Van Hoof E, Aerts I, Meeusen R. Cycling to work: influence on indexes of health in untrained men and women in Flanders. Coronary heart disease and quality of life. Scand J Med Sci Sports. 2008;18(4):498-510

38. Rynders CA, Weltman JY, Jiang B, Breton M, Patrie J, Barrett EJ, et al. Effects of exercise intensity on postprandial improvement in glucose disposal and insulin sensitivity in Prediabetic adults. J Clin Endocrinol Metab. 2014:99(1):220-8.

39. Little JP, Jung ME, Wright AE, Wright W, Manders RJF. Effects of highintensity interval exercise versus continuous moderate-intensity exercise on postprandial glycemic control assessed by continuous glucose monitoring in obese adults. Appl Physiol Nutr Metab. 2014;39(7):835-41.

40. Oja P, Mänttäri A, Heinonen A, Kukkonen-Harjula K, Laukkanen R, Pasanen $M$, et al. Physiological effects of walking and cycling to work. Scand J Med Sci Sports. 1991:1(3):151-7.

41. Hendriksen I, Engbers L, Schrijver J, Gijlswijk RV, Weltevreden J, Wilting J. Elektrisch Fietsen : marktonderzoek en verkenning toekomstmogelijkheden. Leiden: TNO; 2008 2008-01-01. Report No.: 9789059862876.

42. MacArthur J, Cherry C, Harpool M, Daniel S. Electric Boost: Insights from a National E-bike Owner Survey. Portland, OR: Transportation Research and Education Center (TREC); 2018.

43. Schepers JP, Fishman E, den Hertog P, Wolt KK, Schwab AL. The safety of electrically assisted bicycles compared to classic bicycles. Accid Anal Prev. 2014;73:174-80

44. Bassett DRJ, Pucher J, Buehler R, Thompson DL, Crouter SE. Walking, cycling, and obesity rates in Europe, North America, and Australia. J Phys Act Health. 2008;5(6):785-814

\section{Ready to submit your research? Choose BMC and benefit from:}

- fast, convenient online submission

- thorough peer review by experienced researchers in your field

- rapid publication on acceptance

- support for research data, including large and complex data types

- gold Open Access which fosters wider collaboration and increased citations

- maximum visibility for your research: over $100 \mathrm{M}$ website views per year

At BMC, research is always in progress.

Learn more biomedcentral.com/submissions 